\title{
Further investigations of how rare disaster information affects risk taking: A registered replication report
}

\author{
Garston Liang ${ }^{1} \cdot$ Ben Newell ${ }^{1} \cdot$ Tim Rakow $^{2}$ • Eldad Yechiam ${ }^{3}$ \\ Published online: 12 April 2019 \\ (C) The Psychonomic Society, Inc. 2019
}

\begin{abstract}
Across two experiments, Newell, Rakow, Yechiam, and Sambur (Nature Climate Change, 6(2), 158-161, 2016) demonstrated that providing rare disaster information increased people's tolerance for risk-taking. These results motivated a series of as yet-unpublished follow-up experiments involving new manipulations. However, the failure to replicate the original finding in these follow-ups has led our confidence in the original effect to wane. The aim of this registered report was to reconsider the evidence, published and unpublished, for the rare disaster information effect in light of new data. We conducted a large scale replication $(N=242)$ in which we failed to find evidence for the effect reported in Newell et al. thus further reducing our confidence. This registered report format provides a transparent framework by which to address the discrepancy between the published and previously-unpublished findings.
\end{abstract}

Keywords Replication · Disaster information effect · Risky choice

\section{Introduction}

At the close of 2017, the UN Climate Change Conference (COP23) gathered against the backdrop of the most active Atlantic hurricane season in North America. The unprecedented frequency and intensity of hurricanes Harvey, Irma, and Maria warned nations of the risks posed by climateexaggerated natural disasters. These recent events highlight the importance of understanding how individuals interpret and respond to information about the changing environmental risks in the world. The aim of this paper is to evaluate a line of published and unpublished experiments that examined people's reaction to disaster information. Two

Electronic supplementary material The online version of this article (https://doi.org/10.3758/s13423-019-01594-w) contains supplementary material, which is available to authorized users.

Garston Liang

garston.liang@gmail.com

1 School of Psychology, University of New South Wales, Sydney, Kensington, NSW, Australia 2052

2 Institute of Psychiatry, Psychology and Neuroscience, Kings College London, London, UK

3 Faculty of Industrial Engineering and Management, Technion-Israel Institute of Technology, Haifa, Israel experiments in Newell et al. (2016) demonstrated that providing rare disaster information could counter-intuitively increase risk-taking in a computer-controlled micro-world (see Fig. 1).

The micro-world was divided into three villages differentially exposed to natural disasters (see Fig. 1). On each trial, participants chose a house in which to 'live' and received feedback in the form of disaster reports (explained further in Methods). Participants who received the most comprehensive disaster reports that covered the entire micro-world (i.e. forgone feedback for all 3 villages) made riskier choices compared to their less informed counterparts (i.e. forgone feedback for only the selected village, or feedback for only their selected house). By way of the hurricane analogy, informing people about the occurrence of hurricanes in a region resulted in an increase, not decrease, in the likelihood to visit, do business etc. in that disaster-prone region. Despite the paradoxical impact of disaster information on risk taking, confidence in the effect (illustrated in Fig. 2, Panel A) was founded upon several factors; that it replicated across two experimental populations in different countries (total $N=180$ ), persisted over hundreds of rounds of choices, and persisted even when the expected value of the risky choice was worse than that of the safe choice. Moreover, a similar effect of feedback upon risk preference had been found in a simpler risk-taking task involving only monetary gambles (Yechiam, Rakow, \& Newell, 2015). 


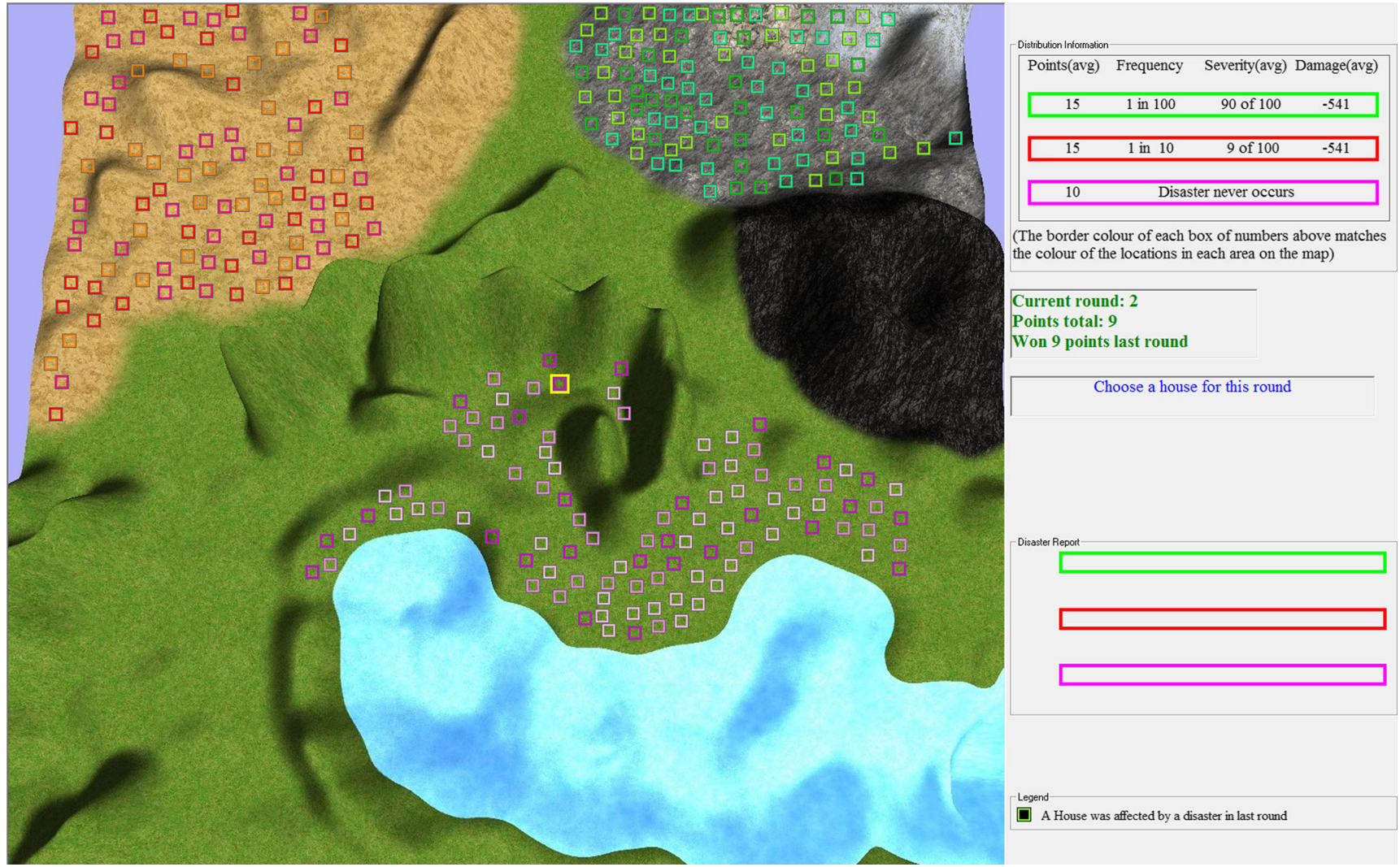

Fig. 1 The micro-world separated into three villages (represented by the three clusters of coloured squares). The top right table displays the payoff distributions for each village and was visible to all participants throughout the experiment (see Table 1 for additional information)

The results of Newell et al. (2016) motivated a series of follow-up experiments to explore why disaster reports covering all the villages produced riskier choices. One explanation was that round-by-round disaster reports with full feedback taught people that 'usually nothing goes wrong', thereby encouraging participants to continually take the risk. To address this explanation, one series of follow-up experiments introduced a historical component to the disaster reports aimed to encourage a long-term view of the risks. By visually depicting the village's disaster history, this manipulation was predicted to reduce the risk-tolerance for participants receiving all-villages disaster reports. A second line of experiments spawned from framing effects in the decisions from experience literature (Hertwig and Erev, 2009; Hertwig, Barron, Weber, \& Erev, 2004). In decisions from experience tasks, a loss framing produces choice patterns consistent with risk seeking (Rakow \& Newell, 2010). Newell et al. (2016) used a mixed framing whereby points were accrued on rounds in which no disaster occurred (analogous to reaping the benefits of a crop harvest in a non-disaster year) and lost due to natural disasters. In this new line of experiments, loss framing was instantiated as round-by-round rental payments. It was predicted that the loss framing would increase the proportion of risky choices.
Ultimately, the original disaster information effect failed to replicate in both lines of experiments (see Fig. 2, Panel B, C and D). Historical elements of the disaster reports did not produce discernible differences in risk taking though the loss framing did increase the overall risky choice proportions. More concerningly however, we failed to replicate the feedback effect in each of the four experiments (replication conditions aggregated in Panel B of Fig. 2; see Supplementary materials for more details). Taken together, after examining the overall risky choice proportions in each of the four follow-up experiments, our confidence in the original feedback effect has waned.

Although it is possible that introducing new manipulations may have influenced the results in unexpected ways, the robustness of the feedback effect is undoubtedly in question. The point of this paper is to not only be candid about our uncertainty, but also to reduce this uncertainty (in one direction or the other). A pre-registered replication offers a transparent framework to examine the stability of the effect, if it exists, while simultaneously avoiding the incentives driving publication bias and file drawers.

Psychology's recent focus on reproducibility has spawned numerous publications on the value of replication and we have adopted some of their recommendations 

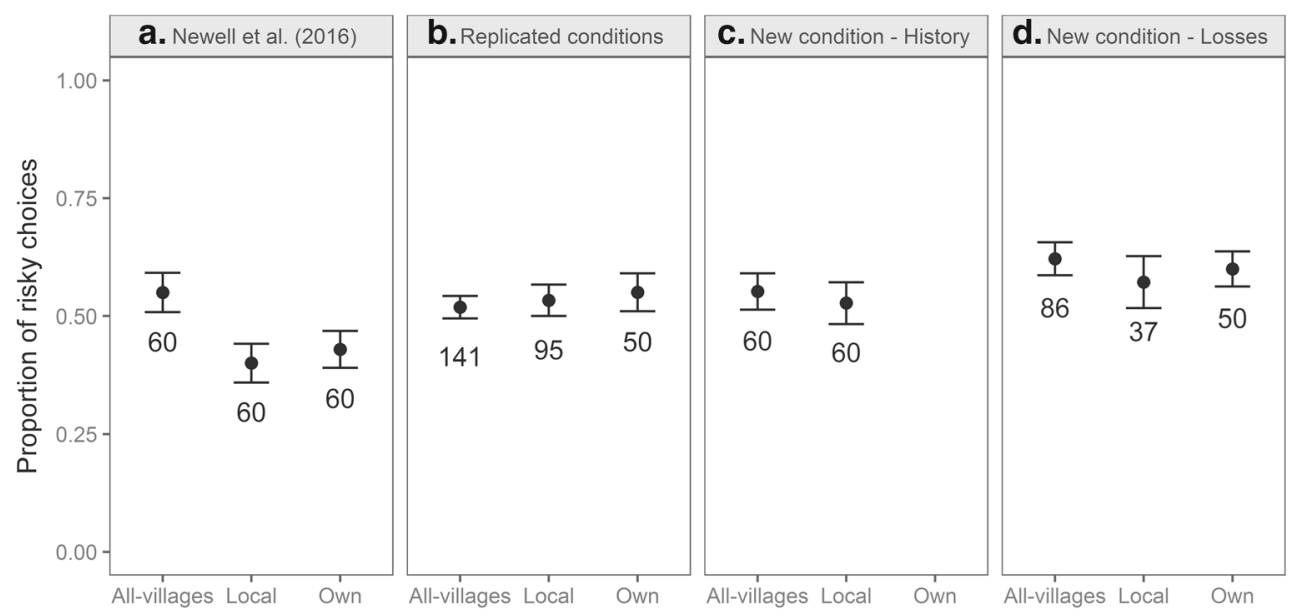

Fig. 2 Risky choice proportion as a function of feedback condition (between-subjects). Error bars depict standard error of the mean and numbers represent N's in each condition. Panel A contains data from the two experiments published in Newell et al. (2016). Panel B contains replication conditions aggregated across four follow-up experiments. Panel C and Panel D each contain data collected from the new conditions (history and losses) of the follow-up unpublished experiments. 'All-villages' refers to receiving feedback about

here (Kruschke, 2013; Simons, 2014; Nosek et al., 2015; Wagenmakers et al., 2017). Principally, we adopt the use of Bayesian statistical tests in this registered replication. Whereas frequentist approaches do not allow for inferences on null results, Bayesian methods allow one to directly quantify evidence for the null hypothesis. One corollary of this is changing the definition of 'success' in a replication from a significant $p$-value to rather accumulating estimates of the effect size (Brandt et al., 2014).

To summarise, the aim of this replication was to reconsider the evidence, published and unpublished, for the rare disaster information effect in light of new data from a pre-approved registered replication. We focused on the comparison of the two between-subjects conditions that showed the largest mean difference in risky choices in the original publication i.e., All-villages and Local village. This choice allowed us to maximize the $n$ in each between-subject condition for the comparison of interest. We hypothesized that rare disaster information covering all the villages would produce a greater proportion of risky choices compared to disaster information covering only the local village.

\section{Methods}

\section{Participants}

In total, 242 participants were recruited from the UNSW undergraduate pool (162 females, $M_{\text {age }}=19.4, S D_{\text {age }}=$ 3.53). Two participants were excluded as they failed to the occurrence of disasters in all of the villages in the micro-world, 'local' refers to receiving feedback only about disasters occurring in the village in which the participant is currently living, and 'own' refers to receiving feedback only about disasters affecting the house currently occupied by the participant (see Fig. 1 for graphical illustration of the micro-world). Follow-up experiment details included in the Supplementary materials

complete the task. Our sampling plan was to collect a minimum of 80 participants in each feedback condition for a total $N$ of 160 . We continued collecting data until either a) model comparisons produced a Bayes factor considered 'strong' evidence for the inclusion or exclusion of the feedback factor, or b) we reached a maximum of 120 participants in each condition. At $N=240$, we discovered one participant was tested in the wrong condition and so to preserve equal $n$ 's in each condition, two additional participants were collected to a total of 242 .

\section{Data}

The data files and pre-registered analyses are available on the Open Science Framework at https://osf.io/zcuhd/.

\section{Materials}

The experiment was displayed at $1920 \times 1080$ resolution, and responses were inputted using a mouse. Participants were presented with a geographical map organised into three villages, each containing 100 houses. The villages were spatially and visually distinct (see Fig. 1). The location, and the associated colour of each village was randomly selected within the program.

On each round, participants selected a house in which to reside from one of three villages (henceforth referred to as 'Safe', 'Risky 1', and 'Risky 2'). The distributions for game points awarded by each village was provided in a table that was available to participants throughout the experiment (see 
top right panel in Fig. 1, and Table 1). In the Safe village, disasters did not occur though the average number of points on each round was modest. The risky villages yielded a more 'attractive' number of points on each round but also bore the risk of a natural disaster, the occurrence of which resulted in a large loss of points. In the Risky 1 village, disasters occurred at a rate of 10 times in 100 rounds, and each disaster affected $9 \%$ of the dwellings in the village. In the Risky 2 village, disasters occurred at a rate of once in 100 rounds though each disaster affected $90 \%$ of dwellings in the village. Therefore, the individual home owner faced the same probability of disasters in both risky regions (see Table 1).

Participants experienced two environments for 200 rounds each: a moderate environment in which all three villages had equal expected value (that is, average number of points per round), and a severe environment in which the two risky regions had a $25 \%$ lower expected value than the safe region (see Table 1). All participants experienced the moderate environment first, followed by the severe environment. Of note, the disaster information effect held across both orderings of the environment (i.e. moderate to severe, and severe to moderate) in Newell et al. (2016).

\section{Design}

Participants were randomly allocated into the two feedback conditions, namely the Local-village, and All-villages feedback conditions. In the Local-village condition, participants received full feedback only for disasters occurring in their chosen village and were able to see which specific houses were damaged. In the All-villages condition, participants received full feedback over the entire micro-world.

\section{Procedure}

Participants were presented with instructions explaining that their task was to select a dwelling in which to reside on each round, and to accrue as many points as possible over the task. Participants were given an accurate description of the risks of a disaster in each region (shown throughout the task in a table in the upper-right of the participants screen; see Table 1) and given feedback following each choice according to their condition. The feedback informed participants whether or not a disaster occurred on that particular round, and the number of points accrued/lost. This round-by-round amount was added to/subtracted from their total point tally which was displayed throughout the task. If participants wished to move houses, a small moving cost was deducted from their point total (see Supplementary materials for more detail). This move cost function was identical to the function in Newell et al. (2016).

Participants first made 200 choices in the 'moderate' environment (four blocks of 50 rounds) before a prompt announced that the environment had changed. The distribution information table (see Table 1) was then updated to reflect the new 'severe' environment and a further 200 choices (four blocks) were made. Participants were debriefed about the aims of the experiment and their remaining points were converted into money at the rate of 1000 points $=\$ 1.10$ AUD.

\section{Results}

The analyses were conducted in RStudio (Version 3.3.3, 2015) using the 'BayesFactor' (Morey \& Rouder, 2018) and 'BEST' packages (Kruschke, 2013).

Table 1 Payoff distributions within each environment

\begin{tabular}{|c|c|c|c|c|c|c|}
\hline & \multicolumn{3}{|c|}{ Moderate environment } & \multicolumn{3}{|c|}{ Severe environment } \\
\hline & Safe & Risky 1 & Risky 2 & Safe & Risky 1 & Risky 2 \\
\hline Payment/round & 10 & 15 & 15 & 10 & 15 & 15 \\
\hline Disaster damage & N/A & -541 & -541 & N/A & -819 & -819 \\
\hline $\mathrm{P}($ disaster $)$ & 0 & 0.01 & 0.10 & 0 & 0.01 & 0.10 \\
\hline $\mathrm{P}($ negative event $\mid$ disaster $)$ & N/A & 0.90 & 0.09 & N/A & 0.90 & 0.09 \\
\hline Therefore, $\mathrm{P}($ negative event $)$ & 0 & 0.009 & 0.009 & 0 & 0.009 & 0.009 \\
\hline Expected value (per round) & +10 & +9.996 & +9.996 & +10 & +7.494 & +7.494 \\
\hline
\end{tabular}

Payment/round is the average amount participants earn per round if no negative events affect their dwelling. Disaster damage is the average amount lost if a negative event affected their dwelling. The variability around each mean outcome was drawn from a uniform distribution of integers, $\mathrm{U}[-3,+3]$. The probability of disaster, $\mathrm{p}$ (disaster), refers to the probability that a disaster would affect a region; the probability of a negative event given that a disaster hits, $\mathrm{P}$ (negative event|disaster), refers to the extent of damage in the region. The information in bold was available to participants; risks were presented as relative frequencies (for example, 1 in 100 rather than 0.01 ; see Fig. 1). N/A, not applicable 
We begin by examining Fig. 3 which plots risky choice proportions within each environment as a function of feedback. The original disaster information effect was that risky choice proportions were greater in the All-villages feedback condition compared to the Local-village feedback condition. The results of this replications indicate the opposite effect; risky choice proportions were higher in the Local-village feedback condition as compared to Allvillages feedback condition. This difference held across both moderate $\left(M_{\text {Local }}=.68, S E=.03\right.$ vs. $M_{\text {All }}=$ $.59, S E=.03)$, and severe environments $\left(M_{\text {Local }}=\right.$ $.54, S E=.03$ vs. $\left.M_{A l l}=.45, S E=.03\right)$. Across feedback conditions, risky choice proportions were lower in the severe environment. This disparity reflected the more punishing loss of points associated with disasters in the severe environment, where the expected value of the Risky villages was $25 \%$ lower than the Safe village. However, the spread of individual-level risky choice proportions across the entire range of values indicate that choices were highly variable. Furthermore, there were a number of individuals at the extremes of 1 and 0 indicating exclusively risky and exclusively the safe choices over the experiment.

Risky choice proportions were analysed using a Bayesian ANOVA with default priors which compared models with factors of feedback, environment, block, and subject as a random factor against a null model of subject alone $\left(\mathrm{H}_{0}\right)$. The all main effects model with feedback, environment, and block was favoured most relative to the null model $\left(B F_{10}=6.66 e+29\right)$. One way to examine how strongly the data support the inclusion of a factor in a model is to examine the inclusion Bayes factor. That is, the relative performance of a nested model that omits the parameter of interest. Comparing the full main effects model to the nested model without feedback $\left(B F_{10}=3.59 e+29\right)$ produced a Bayes factor of 1.85 , anecdotal support for the inclusion of the feedback factor.

The inclusion of the block factor is explained by the gradual decline in risky choice proportions over the experiment. In the All-villages feedback condition, risky choice proportions gradually declined in the initial blocks within the moderate environment before stabilising in the severe environment. By comparison, risky choices in the Local-village condition initially declined at a slower rate followed by a more dramatic drop at the switching of environments (see Supplementary materials for Figure). Both the block and environment effects were consistent with previous experiments (see Supplementary materials for further details).

While the above model comparison analysis can suggest parameters of interest, it does not provide information about the magnitude of these parameters. To more closely examine our main parameter of interest, feedback, we estimated risky choice proportions for the two feedback conditions using Bayesian estimation in the BEST package (Kruschke, 2013). Each feedback group was modelled as a t-distribution with a mean $(\mu)$, standard deviation $(\delta)$, and a shared normality parameter $(v)$ to accommodate outliers. In total, five parameters were estimated using MCMC methods with default, non-committal priors spread widely across the parameter space (priors on the $\mu$ parameters were wide normal distributions, uniform on the $\delta$ parameters, and an exponential on the $v$ parameter). This was to minimize the effect of the prior on the posterior distribution and allow the data to dictate the most credible combination of

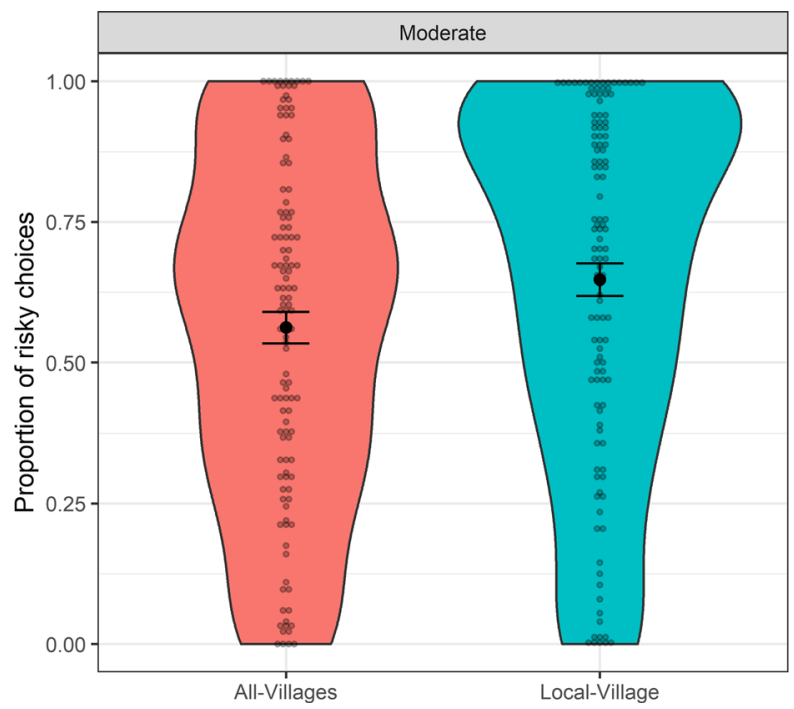

Fig. 3 Violin density plots depict subject-level risky choice proportions as a function of feedback condition (between-subjects) and environment (within-subjects). Points represent individual participants

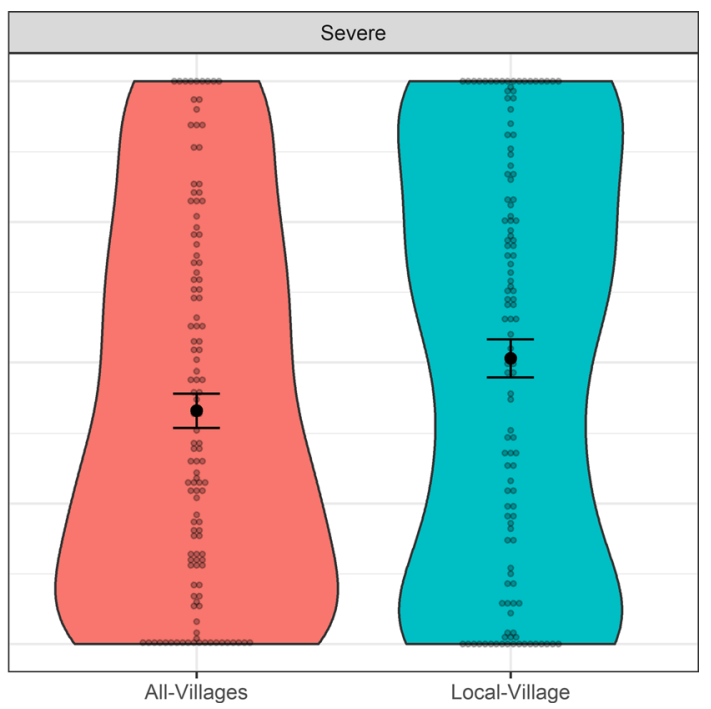

with the mean (solid circle) and standard error also shown. Note that the moderate environment preceded the severe environment 


\section{Difference of Means}

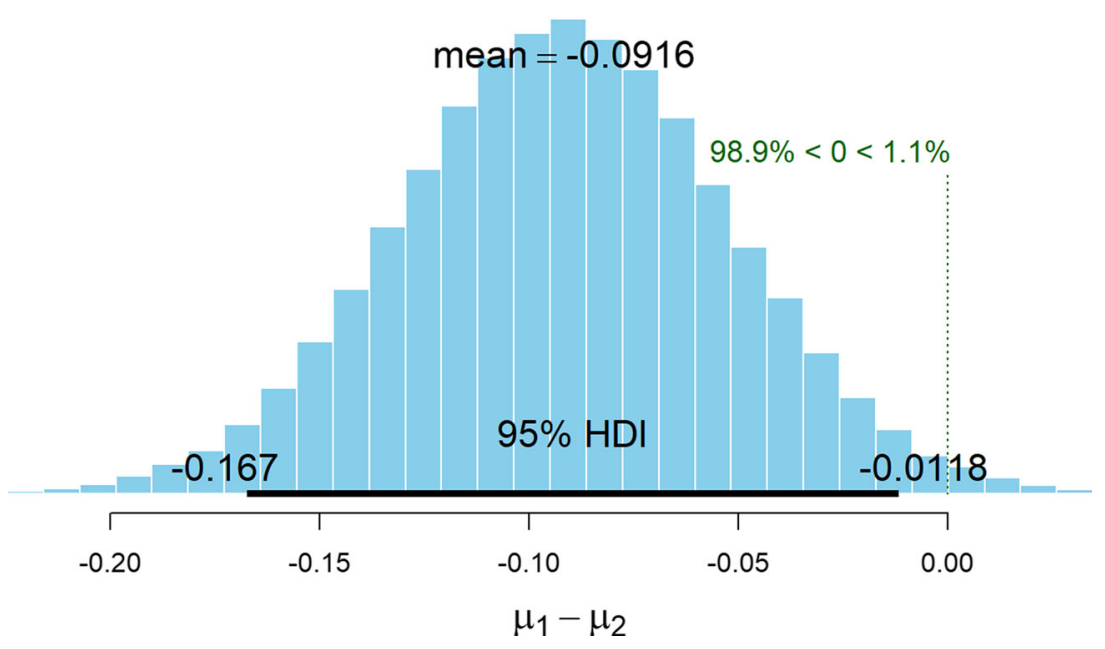

Fig. 4 The distribution of credible differences in risky choice proportion between the feedback conditions. The All-villages condition is $\mu_{1}$ and the Local-village condition is $\mu_{2}$. The $95 \%$ highest density interval ranges from -.167 to -.0118 and notably, $98.9 \%$ of the credible values fall below zero

parameter values (see Kruschke, 2013 for further details). The output of this analysis is shown in Fig. 4 as the posterior distribution for differences between the estimated means $\left(\mu_{1}-\mu_{2}\right)$. The modal difference in risky choice proportions between the All-villages $\left(\mu_{1}\right)$ and Local-village $\left(\mu_{2}\right)$ conditions was -.0916 , with $98.9 \%$ of the credible values falling below zero.

\section{Discussion}

The aim of this paper was to reconsider the evidence for the rare disaster information effect. The results of four unpublished follow-up experiments led our confidence in the effect to wane and this uncertainty was the impetus for conducting a pre-registered replication. As the effects of environment and block were consistent with previous experiments, we focus the discussion on the main parameter of interest, feedback.

The published disaster information effect was the counter-intuitive finding that risk-taking increased with additional disaster related feedback (Newell et al., 2016; Yechiam et al., 2015). The explanation for the effect was that providing rare negative outcomes for a particular region reinforced an attitude that usually 'nothing goes wrong' in that region. However, the effect did not replicate in this registered replication, and - if anything — we find evidence for the opposite effect: more risk taking under feedback for the Local than the All-villages condition. This pattern could now lead to a search for an alternative explanation as to why Local-village feedback produced riskier choices compared to All-village feedback. We are, however, reluctant to pursue such an explanation/speculation at this stage-not least because this is not what we found in our original studies, and to a minimal (non-significant) degree in some of our followup studies (see Introduction). Even in this pre-registered replication, despite an almost $10 \%$ difference in means, evidence for inclusion of feedback in the overall Bayesian model remains anecdotal. Overall, our various examinations of the disaster information effect over the past couple of years suggest that it is fickle: sometimes there is evidence for the original All-villages greater than Local effect, sometimes the opposite, and sometimes neither. Taken together, these results imply that any effect of feedback is small.

However, these datasets and future experiments using this task allow for many other important questions to be asked. For example, how do people react once a disaster has occurred? Does an individual's resistance to moving houses change as a function of witnessing or experiencing the effects of a disaster? Newell et al. (2016) explored some of these questions in their original paper (see Supplementary Materials for that publication), but only in a relatively preliminary way. Indeed, there is recent evidence that decision-makers focus on the sequence of experienced outcomes which can explain a number of probability learning and experience-based phenomena (Plonsky, Teodorescu, \& Erev, 2015, Szollosi, Liang, Konstantinidis, Donkin, \& Newell, in press).

These questions have real life analogues that are pertinent not only to people's reactions to climate disasters but also financial 'shocks', workplace accidents and other adverse outcomes. Investigating how information about these outcomes is provided or represented, and the impact 
of different amounts of information remain worthwhile pursuits. The current experimental setup produces a rich, trial-by-trial data set that would allow one to answer such questions in detail. We look forward to attempting to do so in our future work.

Acknowledgements This study was supported by the Australian Research Council Discovery Project grant (DP160101186).

Author Contributions B.N., T.R., E.Y., and G.L. devised the experiment. G.L. collected the data, conducted the analyses, and wrote the paper. B.N., T.R., and E.Y. edited the final manuscript.

\section{References}

Brandt, M. J., IJzerman, H., Dijksterhuis, A., Farach, F. J., Geller, J., Giner-Sorolla, R., ..., van't Veer, A. (2014). The replication recipe: What makes for a convincing replication Journal of Experimental Social Psychology, 50(1), 217-224.

Hertwig, R., Barron, G., Weber, E. U., \& Erev, I. (2004). Decision from experience and the effect of rare events in risky choice. Psychological Science, 15(8), 534-539.

Hertwig, R., \& Erev, I. (2009). The description-experience gap in risky choice framing. Trends in Cognitive Sciences, 13(12), 517-523.

Kruschke, J. K. (2013). Bayesian estimation supersedes the t test. Journal of Experimental Psychology: General, 142(2), 573.

Morey, R. D., \& Rouder, J. N. (2018). BayesFactor: Computation of Bayes factors for common designs BayesFactor. R package version 0.9.12-4.2. https://CRAN.R-project.org/ package $=$ BayesFactor.
Newell, B. R., Rakow, T., Yechiam, E., \& Sambur, M. (2016). Rare disaster information can increase risk-taking. Nature Climate Change, 6(2), 158-161.

Nosek, B. A., Alter, G., Banks, G. C., Borsboom, D., Bowman, S. D., Breckler, S. J., ..., Yarkoni, T. (2015). Promoting an open research culture. Science, 348(6242), 1422-1425.

Plonsky, O., Teodorescu, K., \& Erev, I. (2015). Reliance on small samples, the wavy recency effect, and similarity-based learning. Psychological Review, 122(4), 621-647.

Rakow, T., \& Newell, B. R. (2010). Degrees of uncertainty: An overview and framework for future research on experiencebased choice. Journal of Behavioral Decision Making, 23(1), 114.

Simons, D. J. (2014). The value of direct replication. Perspectives on Psychological Science, 9(1), 76-80.

Szollosi, A., Liang, G., Konstantinidis, E., Donkin, C., \& Newell, B. R. (in press). Simultaneous underweighting and overestimation of rare events: Unpacking a paradox. Journal of Experimental Psychology: General.

Wagenmakers, E.-J., Verhagen, J., Ly, A., Matzke, D., Steingroever, H., Rouder, J. N., \& Morey, R. D. (2017). The need for Bayesian hypothesis testing in psychological science. In S. O. Lilienfeld, \& I. D. Waldman (Eds.), Psychological science under scrutiny: Recent challenges and proposed solutions (pp. 123-138). WileyBlackwell.

Yechiam, E., Rakow, T., \& Newell, B. R. (2015). Superunderweighting of rare events with repeated descriptive summaries. Journal of Behavioral Decision Making, 28(1), 67-75.

Publisher's note Springer Nature remains neutral with regard to jurisdictional claims in published maps and institutional affiliations. 